\title{
ROBUST PATH RECONSTRUCTION IN LARGE SCALE WIRELESS SENSOR NETWORKS
}

\author{
Nida Afreen L M ${ }^{1}$, Umashankar $M^{2}$ \\ ${ }^{I}$ M.Tech Research Scholar, Deptartment of CSE, SJB Institute of Technology, Bangalore, India \\ nida.afreen18@gmail.com \\ ${ }^{2}$ Assistant Professor, Department of CSE, SJB Institute of Technology, Bangalore, India \\ ums.gowda@gmail.com
}

\begin{abstract}
The demand for the compact and small-sized electronics device is increasing in present days, and many of the organizations and researchers have adopted these demand and have succeeded too. Wireless sensor devices are made with more compact and small sized and also the large scale Wireless Sensor Networks (LSWSNs), which are also developed with more number of sensor nodes. These LSWSNs means the WSNs were having high density or of the large area of the network. Mainly a WSNs are composed of Sensor Nodes (SNs) which are placed in a pattern, by which more data can be gathered and transferred to the base station (BS) or also known as Sink. The path determining process from source to destination for data transmission is generally known as routing. In most WSNs routing of incoming data can be determined by the network layer. The SNs at the source cannot reach the sink node directly in multi-hop networks, hence intermediate SNs need to relay their respective packets. Mainly the routing path of every packet is helpful in understanding the network performance. The analysis of routing path is necessary to route path reconstruction. There have been many efforts made to address the per-packet path reconstruction problem in WSNs. In this project we propose a novel Pathfinder scheme. The evaluation will be done using Matlab tool.
\end{abstract}

Keywords: Sensor Node, WSNs, Power optimization, Path Reconstruction, Path finder.

\section{INTRODUCTION}

Wireless Sensor Network it is a geographically, randomly distributed network of sensor nodes connected using adhoc technology. Where the data measured at each node is assumed to be delivered at a common collected node called a Sink Node, from where through a gateway the aggregated data could reach to the monitoring stations for a specific application. Sensor is an electronic device. Which is used for measuring the changes into environ[1]. It is available in lower cost and enough research has been conducted to minimize the size of it, so today we have very cheaper and smaller sensor available, even on dust size. Sensor node is a computing device, which takes environ change as an input by its subunit called sensory unit (where the measured data is converted into electrical signal), this signal is further processed by its micro-controller unit (which will have typically, real-time operating system (RTOS), buffer and storage memory and processing unit), then the processed signal is further communicated to another such node in vicinity through a subunit called communication unit wirelessly.

The path determining process from source to the sink of data transmission is known as Routing. In WSNs, the routing of incoming data is performed with the network layer. Commonly a source node doesn't link the sink node directly in case of multi-hop networks. Hence, there is need of intermediate SNs to relay their respective packets [2]. A routing path will help out to solve this issue. Reconstruction of routing path is the biggest task in routing algorithm. Most of the sensors are needed to deliver the messages successfully between source and destination. Although there is the need for some applications that should provide the assurance of it, this is considered as the real-time application requirement for message delivery and also maximizes the network lifetime [3]. The message delivery assurance for entire routing protocol is indispensable, means the protocol should need be find any routing path for the communication nodes even if the route does not exist. The assurance is proven in the formula while the ratio of message delivery can be used for average case performance evaluation. Some of the applications require the perfect delivery in the specified time or else the message will be considered as the useless or after the time limit, the information content will be decreased. Thus, some routing protocols mainly perform for delay time control of the network [6,7]. The average performance these type of protocols is evaluated by measuring the message delivery accordingly with time factors. Some routing protocols have adopted their main objectives for network lifetime. These protocols also aim to balance or reduce the energy consumption among the nodes by considering their energy levels. The metric is used for lifetime network determination, which is application dependent. Most of the protocols consider the every node is important equally from starting time of node to die time as a metric or energy consumption of every node [8].

The proposed system have a novel path finder and path reconstructing method and also data compression scheme to optimize the power in very large wireless sensor networks. 
Section 2 discusses about the related work followed by proposed system in Section 3. Section 4 discusses about the proposed system design section 5 gives the outcomes of the study, while Section 5 makes concluding remarks.

\section{RELATED WORK}

This section gives the brief literature survey of the many researchers who have carried their study and experiments on LSSNs and in routing path reconstruction. The research work is divided into two categories like the contribution for Large-scale sensor networks and contribution for routing path reconstruction.

Khlif et al. [9] have presented a mechanism for set representation of the casual order in Large scale distributed system. The mechanism is based on the immediate dependency relation to having automatic model of any large scale distributed system. The model resultants can be represented only in graph form. The graph is used for designing the efficient algorithms, verification, and validation, etc. This mechanism is implemented in $\mathrm{C}++$. The study execution outcomes with a viability of support towards large scale systems. Stamatescu et al. [10] have discussed architecture for large scale heterogeneous monitoring system to have sensor fusion. The authors focused on the communication and data processing integration of WSNs. The study outcome with the realworld applications of UAV platform and also in the ground sensor network subsystem.

Zhu et al. [11] have provided the study on the average energy consumption in LSWSNs of sensor node distribution in hexagon manner. Monte Carlo method is being used to verify and simulated the distance distribution function and average energy consumption function. The simulation results with closer calculation results of both theoretical and calculated results. The energy consumption is being calculated by using this method. Samarasinghe and Leone [12] have presented the scalable routing in LSWSNs (ad-hoc networks) and is known as Greedy routing. The routing zone divides the network into zones of geographical coordinates. The study outcomes the ability to adopt in a tree-based protocol to attain improved performance and scalability.

Hu and Wang [13] have presented the heuristic algorithm to reduce the number of mobile chargers in rechargeable LSWSNs. The authors have focused on the problem of charging of SNs continuously. The author has used two steps for solving the above issue. The simulation results from outcome with the average bound of <1.1. Casini et al. [14] have given the innovative approach for the integration of human machine in decision making in LSWSNs, which rely on Hadoop Map Reduce framework.

Wang et al. [15] have illustrated the novel localization scheme for LSWSNs with anchor nodes. The scheme is helpful in reduction of anchor nodes cost. By using the modified algorithms of Floyd and anchor nodes, better performance can be achieved and is valid out by analytical and simulation results. Salmins et al. [16] have presented a method to solve the software related issues of LSWSNs testbed. The experiment results outcome with advantageous results for more than $20 \mathrm{SNs}$ in LSWSNs and efficiency rapidly increases with the number of SNs testbed increment.

Dulman and Pauwels [17] has provided a Tweaking Gosip algorithm for LSWSNs computations. The study outcomes with the robust aggregation method. Further study of the author has discussed the performance-based mechanism.

Gao et. al. [18] have presented the Pathfinder for reconstruction of the routing path. The authors have studied the issues of existing approaches to path reconstruction. The path finder helps in the exploitation of temporal correction between the packet paths set and path information compression. With the simulation results the study outcomes with the high reconstruction ratio and also low transmission overhead.

Liu et al. [19] have presented a path reconstruction method by using the compressive sensing in Dynamic WSNs. The experiment results in simulation give the high accuracy in path recovery of $100 \%$ and outperform the state of art approaches with 96\%. Gao et al. [20] have presented Pathfinder against the losses of the routing dynamics. The author also presented an analytical model to analyze the Pathfinder performance. The experimental results show that the Pathfinder provides the low transmission cost and high reconstruction ratio compared with existing methods.

\section{PROBLEM STATEMENT}

"To design and develop a mechanism to re-construct path for each packet with minimal possible overhead to achieve the study of routing path of packets in large scale wireless sensor network by building an algorithm using temporal correlation among local packets computing path differences."

\section{PROPOSED MODEL}

The prime purpose of the proposed system is to perform power optimization in very large WSN and also path reconstruction of the system. Pathfinder comprises of two primary segments for way recreation as appeared in above figure. At the hub side, the way recording part abuses worldly connection among an arrangement of bundle ways and productively packs the way data utilizing way distinction. At the PC side, the way reproduction segment deduces parcel ways from the compacted data and utilizes astute way theory to recreate the bundle ways with high remaking proportion. At the node side, path difference of each packet is recorded in three data structures, bit vector, container and XOR-byte which are updated hop-by-hop. 


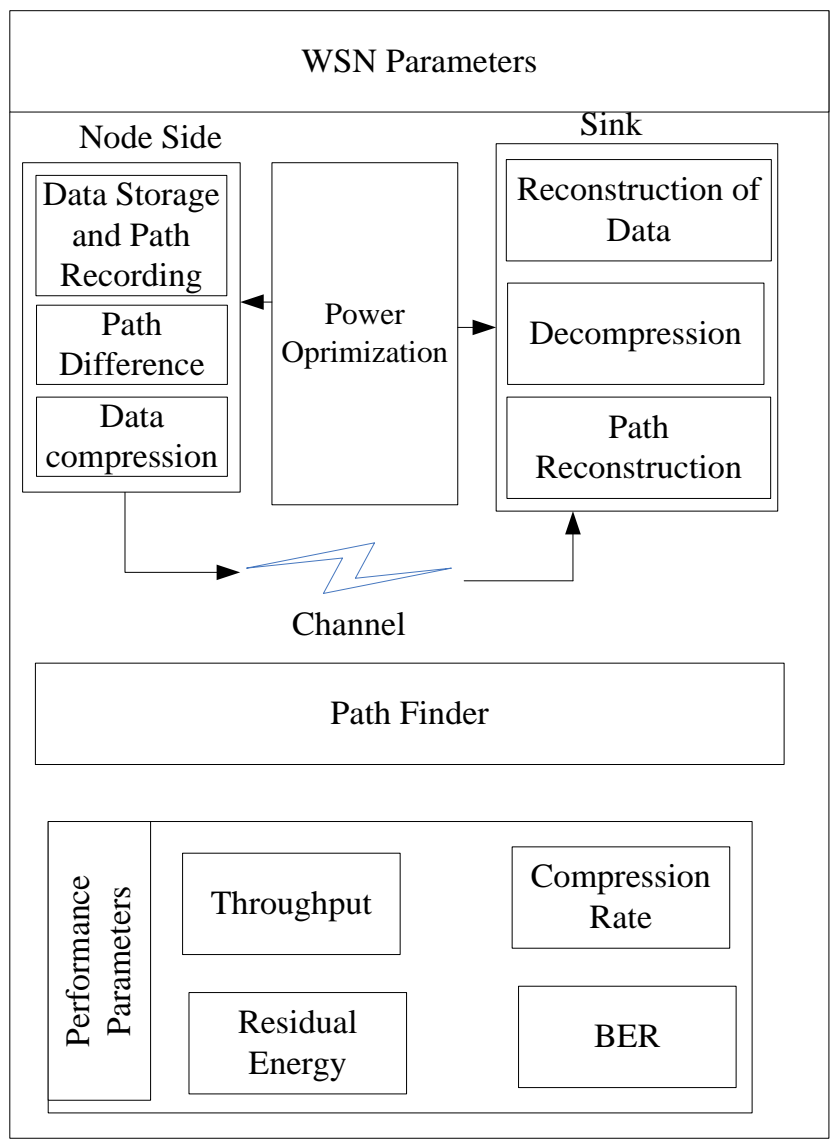

Fig.3 Proposed system architecture

Figure.3 illustrates how path information of packets is updated at node fi. The XOR-byte XORs all the least significant bytes of the travelled nodes IDs together. It is used to verify whether a reconstructed path is correct or not. A key observation revealed in Section II shows that although there exists relatively high loss rate and routing dynamics in real-world large scale sensor networks, the path Difference, however, is usually small, e.g., $\leq 2$.

We proposed a robust path reconstruction technique using in very large wireless sensor network. It optimizes the power in the entire network. Here we are using data compression method to compress the data in the node, then we are using a path recording method and also the path reconstruction techniques. The recent advancement in the technology has made feasible for manufacturing the small and low-cost sensors. The sensing devices measure the environmental conditions and transfer them into the electrical signal. This signal processing will help out in identifying the object location or event happening etc. A large number of sensors are networked in many applications to identify the unattainable operation. WSNs are consist of thousands of sensors, which provides the communication between the Source and BS/sink. A greater number of sensors allows for sensing over larger geographical regions with greater accuracy.

\section{RESULT ANALYSIS}

This proposed study considers a robust path reconstruction technique in very large scale wireless sensor networks. The simulation in done using Matlab tools. In this simulation, we initialized some parameters like network scale is 300, then deployed a network. The base station coordinates also initialized for both XY-coordinates to deploy the base station in the network. After deployment of the base station, we created a reference link from base station to all sensor nodes the sends data form sensor nodes to base station. Then we initialized lambda value is 10 , and loss rate is 0.1 . Then we executed a reference path detection algorithm. Then finally performed a communication between base station and all the sensor nodes. Fig. 6.1 shows deployment of sensor network.

Fig. 6.4 shows the reference path created form sensor node 1 to the base station. This communicating the data through reference path like 1, 22, 203, 165, 42 and BS from sensor node 1 to the base station.

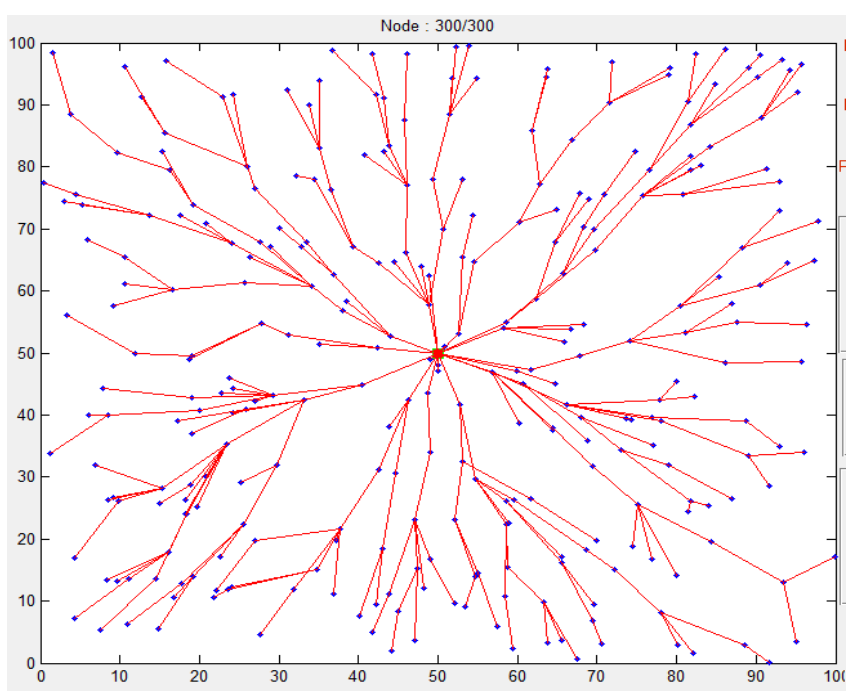

Figure. 6.3 Create reference links

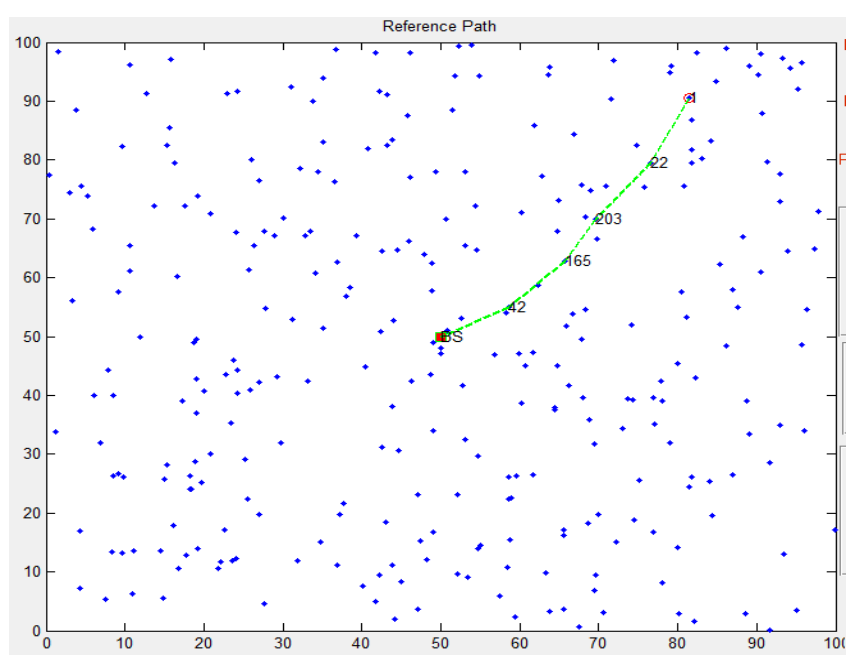

Figure. 6.4 shows reference path from node-1. 


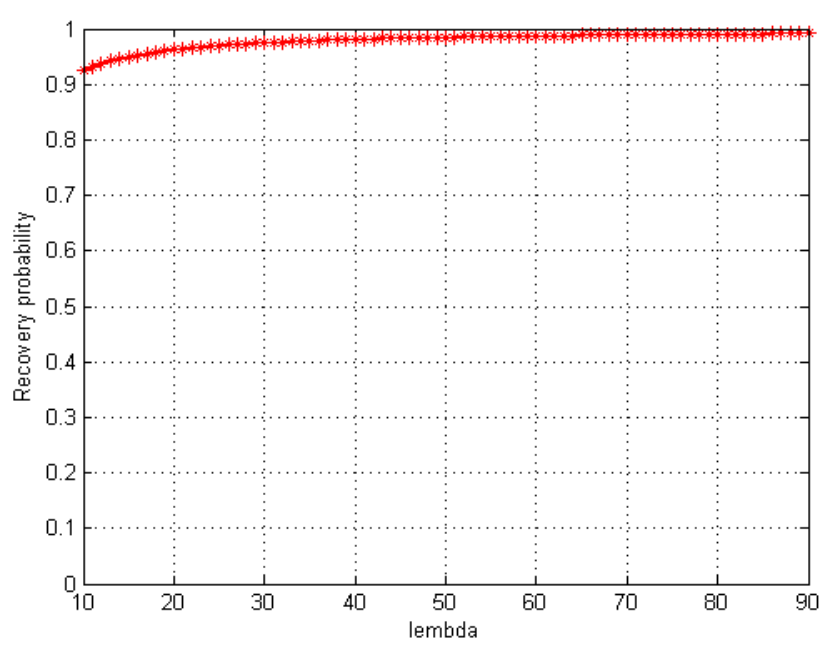

Fig 6.5 Recovery probability vs. lambda.

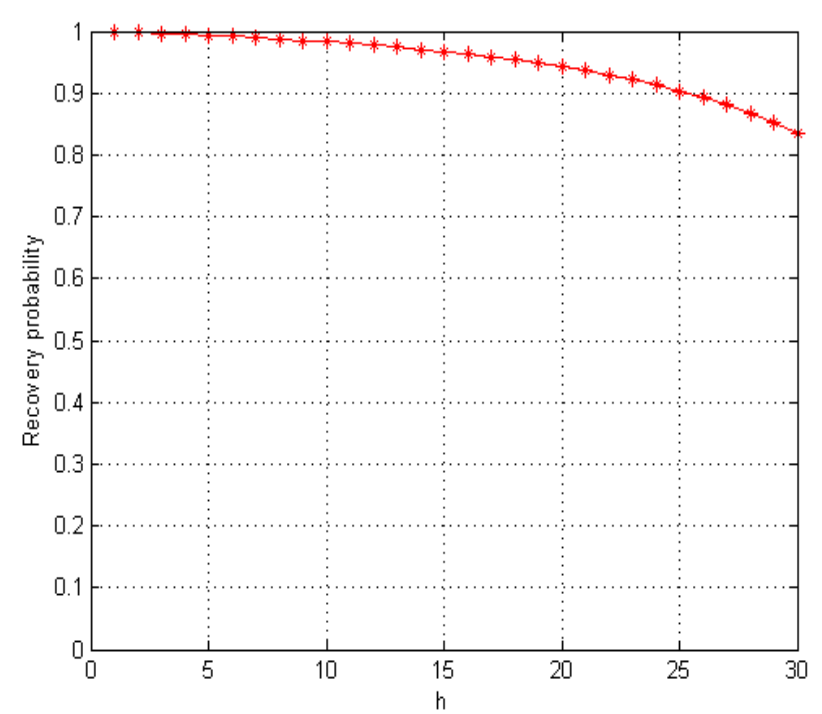

Fig. 6.6 Recovery probability vs. h

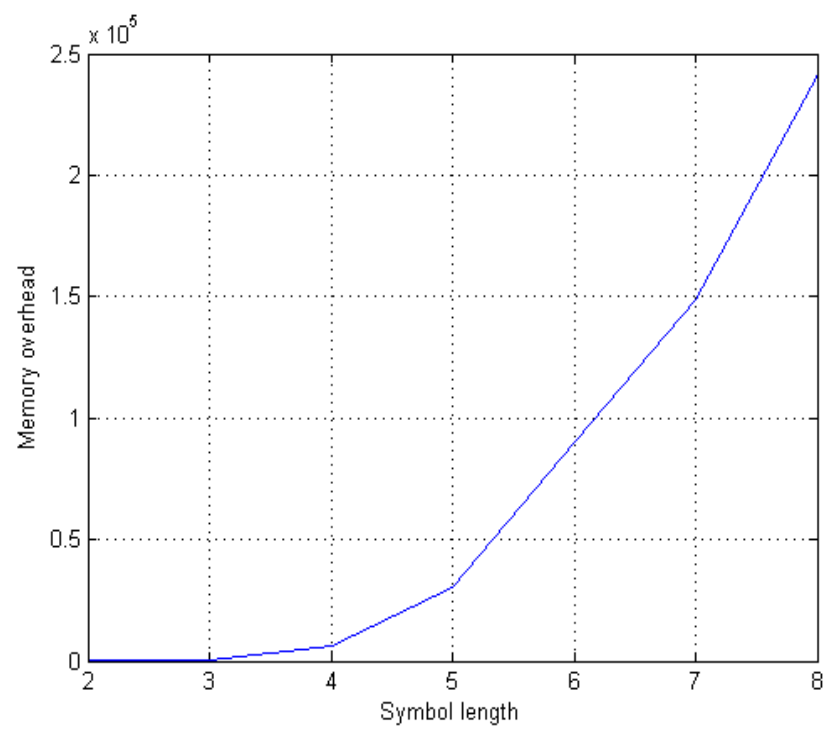

Fig. 6.8 Memory overhead vs. symbol length.

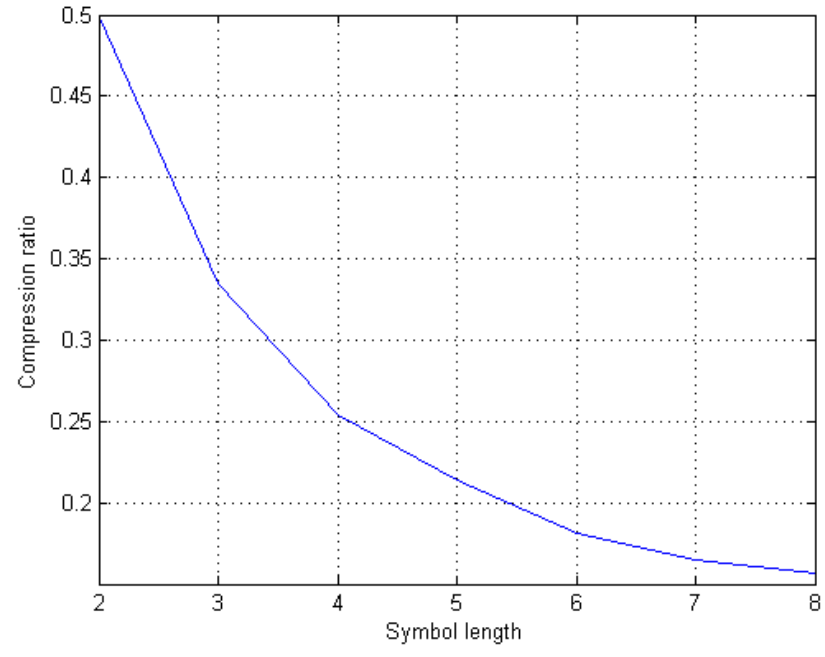

Fig. 6.9 Compression ratio vs. symbol length.

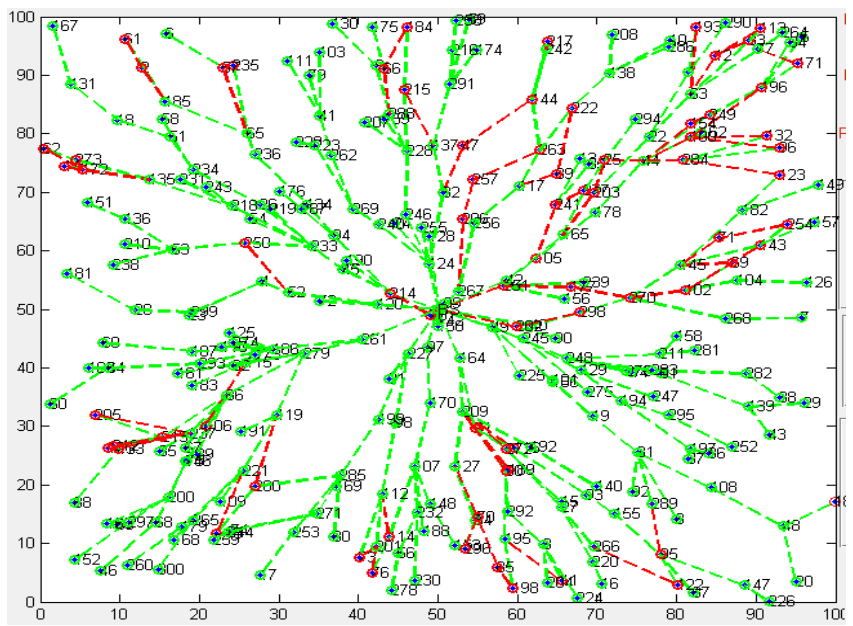

Fig. 6.10 Path finding done for all the nodes.

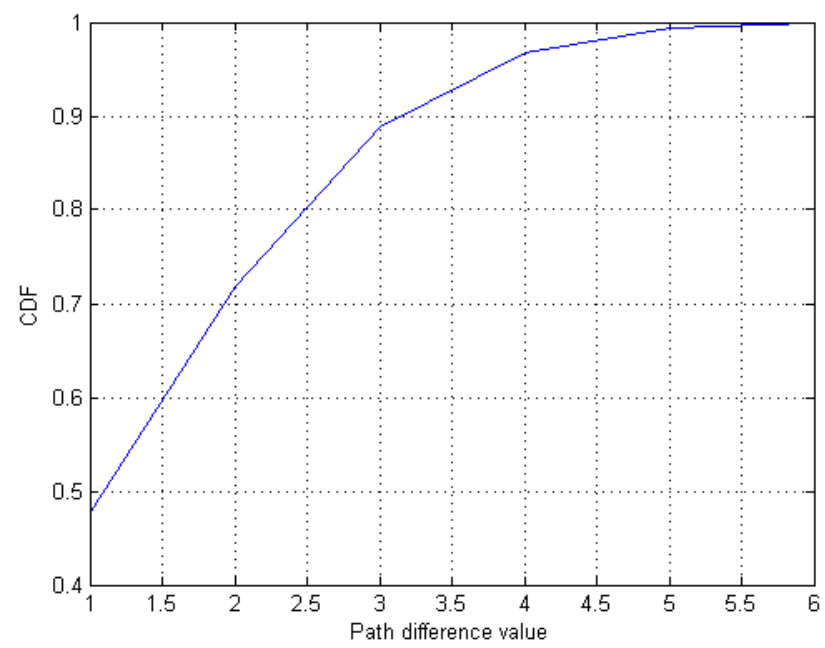

Fig. 6.14 CDF vs. length of bit vector.

The performance communication between sensor node- 1 to the bases station, is calculating the distance with center path is $122 \quad 203 \quad 165 \quad 42$ 301. Then, after this reference path, the routing path will be found is $1,22,203,165,42$, to base station. The path bit vector also calculated is 11111 . Fig. 6.5 shows the recovery probability vs. lambda. Here we 
can observe that, the as the lambda value increases, the recovery probability is almost equals to 1 . Then fig. 6.6 give the recovery probability vs. $h$ value. Here also we can observe that, the recovery probability increases, the $h$ value is slightly decrease, both $h$ value and lambda value are inversely proportional.

\section{CONCLUSION AND FUTURE WORK}

The recent advancement in the technology has made feasible for manufacturing the small and low-cost sensors. The sensing devices measure the environmental conditions and transfer them into the electrical signal. This signal processing will help out in identifying the object location or event happening etc. A large number of sensors are networked in many applications to identify the un-attainable operation. WSNs are consist of thousands of sensors, which provides the communication between the Source and BS/sink. A greater number of sensors allows for sensing over larger geographical regions with greater accuracy. Many issues in routing and design challenges in WSNs are listed and briefly explained.

\section{REFERENCE}

[1] Bulusu, Nirupama, John Heidemann, and Deborah Estrin. "GPS-less low-cost outdoor localization for very small devices." Personal Communications, IEEE 7.5 (2000): 28-34.

[2] Sohraby, Kazem, Daniel Minoli, and Taieb Znati. Wireless sensor networks: technology, protocols, and applications. John Wiley \& Sons, 2007.

[3] Ahn, Gahng-Seop. Information-driven tracking and access control in wireless ad hoc and sensor networks. Diss. Columbia University, 2009.

[4] Sharma, Gaurav. Routing in wireless sensor networks. Diss. THAPAR UNIVERSITY PATIALA, 2009.

[5] Labrador, Miguel A., and Pedro M. Wightman. Topology Control in Wireless Sensor Networks: with a companion simulation tool for teaching and research. Springer Science \& Business Media, 2009.

[6] Acs, Gergely, and Levente Buttyán. "A taxonomy of routing protocols for wireless sensor networks." BUTE Telecommunication department (2007).

[7] Li, Xiangfang. Multi-layer Optimization in Wireless Ad Hoc Networks. ProQuest, 2007.

[8] Boukerche, Azzedine, et al. "Routing protocols in ad hoc networks: A survey." Computer Networks 55.13 (2011): 3032-3080.

[9] Khlif, H.; Hadj Kacem, H.; Pomares Hernandez, S.E.; Hadj Kacem, A., "A Mechanism for the Causal Ordered Set Representation in Large-Scale Distributed Systems," in Enabling Technologies: Infrastructure for Collaborative Enterprises (WETICE), 2015 IEEE 24th International Conference on , vol., no., pp.23-28, 15-17 June 2015.

[10] Stamatescu G.; Stamatescu, I.; Dragana, C.; Popescu, D., "Large-scale heterogeneous monitoring system with decentralized sensor fusion," in Intelligent Data Acquisition and Advanced Computing Systems: Technology and Applications (IDAACS), 2015 IEEE 8th International Conference on, vol.1, no., pp.2-5, 2426 Sept. 2015.

[11] Haofu Zhu; Jianlin Mao; Le Wang; Lixia Fu; Ning Guo, "The study on point average energy consumption by Monte Carlo in large-scale wireless sensor networks," in Information and Automation, 2015 IEEE International Conference on, vol., no., pp.1700-1703, 810 Aug. 2015.

[12] Samarasinghe, K.; Leone, P., "Greedy Zone Routing: Scalable routing in large scale wireless ad-hoc networks," in Sensing, Communication, and Networking (SECON), 2015 12th Annual IEEE International Conference on, vol., no., pp.172-174, 2225 June 2015.

[13] Cheng $\mathrm{Hu}$; Yun Wang, "Minimizing the number of mobile chargers in a large-scale wireless rechargeable sensor network," in Wireless Communications and Networking Conference (WCNC), 2015 IEEE, vol., no., pp.1297-1302, 9-12 March 2015.

[14] Casini, E.; Depree, J.; Suri, N.; Bradshaw, J.M.; Nieten, T., "Enhancing decision-making by leveraging human intervention in large-scale sensor networks," in Cognitive Methods in Situation Awareness and Decision Support (CogSIMA), 2015 IEEE International Inter-Disciplinary Conference on , vol., no., pp.200205, 9-12 March 2015.

[15]Xizhe Wang; Jian Qiu; Jin Fan; Guojun Dai, "MDSbased localization scheme for large-scale WSNs within sparse anchor nodes," in Communications (ICC), 2015 IEEE International Conference on, vol., no., pp.66096614, 8-12 June 2015.

[16] Salmins, A.; Ozols, K.; Ruskuls, R., "Data management in TestBed for large-scale wireless sensor networks," in Advances in Wireless and Optical Communications (RTUWO), 2015, vol., no., pp.54-57, 5-6 Nov. 2015.

[17]Dulman, S.; Pauwels, E., "Tweaking gossip algorithms for computations in large-scale networks," in Intelligent Sensors, Sensor Networks and Information Processing (ISSNIP), 2015 IEEE Tenth International Conference on, vol., no., pp.1-6, 7-9 April 2015.

[18] Yi Gao; Wei Dong; Chun Chen; Jiajun Bu; Gaoyang Guan; Xuefeng Zhang; Xue Liu, "Pathfinder: Robust path reconstruction in large scale sensor networks with lossy links," in Network Protocols (ICNP), 2013 21st IEEE International Conference on , vol., no., pp.1-10, 710 Oct. 2013.

[19] Gao, Y.; Dong, W.; Chen, C.; Bu, J.; Wu, W.; Liu, X., "iPath: Path Inference in Wireless Sensor Networks," in Networking, IEEE/ACM Transactions on, vol.PP, no.99, pp.1-1, 2014.

[20]Liu, Z.; Li, Z.; Li, M.; Xing, W.; Lu, D., "Path Reconstruction in Dynamic Wireless Sensor Networks Using Compressive Sensing," in Networking, IEEE/ACM Transactions on, vol.PP, no.99, pp.1-1, 2015 . 